Tôhoku Math. Journ.

38 (1986), 469-490.

\title{
INTERPOLATION OF OPERATORS IN LEBESGUE SPACES \\ WITH MIXED NORM AND ITS APPLICATIONS TO FOURIER ANALYSIS
}

\author{
SATORU IGARI* \\ (Received December 24, 1985)
}

1. Intoroduction. In this paper we show interpolation theorems for linear operators in Lebesgue spaces with mixed norm and apply them to Fourier analysis.

Let $M$ be a measure space and $M^{m}$ be an $m$ product space $\prod_{j=0}^{m-1} M_{j}$ where $M_{j}$ are copies of $M$. Let $L^{t}\left(L^{s}\right)=L^{t}\left(M^{n} ; L^{s}\left(M^{m}\right)\right)$ be a Lebesgue space with mixed norm $\left(\int_{M^{n}}\left(\int_{M^{m}}|f|^{s}\right)^{t / s}\right)^{1 / t}$. Let $T$ be a linear operator in $L^{t}\left(L^{s}\right)$. Under the assumption that $T$ is bounded in $L^{t}\left(L^{s}\right)$ for every permutation of $\left\{M_{j} ; j=0,1, \cdots, d-1\right\}$, we discuss the boundedness of $T$ in the space $L^{u}\left(M^{m+n}\right)$, where

$$
1 / u=(m / s+n / t) /(m+n) .
$$

Part I deals with interpolation problem. In $\S 2$ we introduce auxiliary holomorphic functionals $W^{z}$ and $F^{z}$ in $|z|<1$. We divide the unit circle into several arcs and estimate these functionals by $L^{t}\left(M^{n} ; L^{8}\left(M^{m}\right)\right)$-norm for $z$ in an arc, where the choice of permutation of the measure spaces $\left\{M_{j}\right\}$ depends on the arc. This is the idea to prove our interpolation theorems and they are given in $\S 3$. To get bounds of the functionals we restrict the domain of $F^{z}$ to functions in $\prod_{j=0}^{m+n-1} L^{u}\left(M_{j}\right)$. As a consequence the domain of the linear operators of Remark 1 and Theorem 3 in $\S 3$ are restricted to $\Pi L^{u}\left(M_{j}\right)$, but this condition is unremovable (cf. Remark 4 in §5).

In Part II we shall apply our interpolation theorems to two problems in Fourier analysis which are closely related. In $\S 4$ we consider the Riesz-Bochner summing opertor $s^{\varepsilon}, \varepsilon>0$. For a function $f$ on the $d$ dimensional Euclidean space $\boldsymbol{R}^{d}$ the Riesz-Bochner mean $\boldsymbol{s}^{\varepsilon}(f)$ of order $\varepsilon$ is defined by

$$
s^{\varepsilon}(f)^{\wedge}(\xi)=\left(1-|\xi|^{2}\right)^{\varepsilon} \hat{f}(\xi)
$$

for $|\xi|<1$ and $=0$ otherwise, where $\hat{f}$ is the Fourier transform of $f$ :

\footnotetext{
* Partly supported by the Grant-in-Aid for Scientific and Co-operative Research, the Ministry of Education, Science and Culture, Japan.
} 


$$
\hat{f}(\xi)=\frac{1}{\sqrt{2 \pi}{ }^{d}} \int_{R^{d}} f(x) e^{-i \xi x} d x
$$

For $d>1$ the operator $s^{0}$ is not $L^{p}$-bounded if $p \neq 2$ (Fefferman [5]) but $s^{0}$ is $L^{p}$-bounded if it is restricted to radial functions and if $2 \mathrm{~d} /(d+1)<$ $p<2 d /(d-1)$ (Herz [8]). For $d=2, \varepsilon>0$ and $4 / 3 \leqq p \leqq 4$, the operator $s^{\varepsilon}$ is $L^{p}$-bounded (Carleson and Sjolin [2]). Later, several proofs of the Carleson-Sjölin theorem were given (Hörmander [9], Fefferman [6] and Cordoba [4]).

In $\S \S 4.1-4.4$ we shall prove that if $\varepsilon>0$

$$
\int_{\boldsymbol{R}^{2}}\left(\int_{\boldsymbol{R}^{d-2}}\left|s^{\varepsilon}(f)\right|^{2} d x(p)\right)^{4 / 2} d x(\complement p) \leqq \mathrm{const} \int_{\boldsymbol{R}^{2}}\left(\int_{\boldsymbol{R}^{d-2}}|f|^{2} d x(p)\right)^{4 / 2} d x(\complement p)
$$

for all reasonable function $f$ on $\boldsymbol{R}^{d}$, where $x(p)=\left(x_{0}, \cdots, x_{d-3}\right)$ and $x(\mathfrak{C} p)=$ $\left(x_{d-2}, x_{d-1}\right)$. Since the operator $s^{\varepsilon}$ is rotation invariant (1.1) holds for any permutation of variables $\left(x_{0}, x_{1}, \cdots, x_{d-1}\right)$. Therefore our interpolation theorem applies to $s^{\varepsilon}$ and we get the inequality $\left\|s^{\varepsilon}(f)\right\|_{p} \leqq$ const $\|f\|_{p}$ if $\varepsilon>0,2 d /(d+1) \leqq p \leqq 2$ and if $f$ is of the form $f_{0}\left(x_{0}\right) f_{1}\left(x_{1}\right) \cdots f_{d-1}\left(x_{d-1}\right)$.

In $\S 5$ we consider the restriction problem of Fourier transform.

In the following, $C$ will denote constants which may be different in each occasion and $\mathscr{S}\left(\boldsymbol{R}^{d}\right)$ the set of functions in $\boldsymbol{R}^{d}$ infinitely differentiable and rapidly decreasing.

\section{Part I. Interpolation of operators.}

2. Notations and auxiliary functions. Let $(M, \mathscr{A}, \mu)$ be a $\sigma$-finite measure space. Let $d$ be a positive integer and $\left(M_{j}, \mathscr{M}_{j}, \mu_{j}\right), j=0,1, \cdots$, $d-1$, be copies of $(M, \mathscr{M}, \mu)$. Let $(\bar{M}, \overline{\mathscr{M}}, \bar{\mu})$ be the product measure space $\prod_{j=0}^{d-1}\left(M_{j}, \mathscr{L}_{j}, \mu_{j}\right)$. For a subset $p=\left\{p_{0}, p_{1}, \cdots, p_{m-1}\right\}$ of $\{0,1, \cdots, d-1\}$ we denote $(M(p), \mathscr{M}(p), \mu(p))=\Pi_{j=0}^{m-1}\left(M_{p_{j}}, \mathscr{M}_{p_{j}}, \mu_{p_{j}}\right)$. For a subset $p$ of $\{0,1, \cdots, d-1\}$ denote $\complement p=\{0,1, \cdots, d-1\}-p$. Thus $d \mu(p)\left(x_{p_{0}}, \cdots\right.$, $\left.x_{p_{m-1}}\right)=d \mu_{p_{0}}\left(x_{p_{0}}\right) \cdots d \mu_{p_{m-1}}\left(x_{p_{m-1}}\right)$ and $d \mu(p) \times d \mu(\complement p)=d \bar{\mu}$.

For $1 \leqq s<\infty L^{s}(\bar{M})$ denotes the Lebesgue space with norm $\|f\|_{s}=$ $\left(\int_{\bar{M}}|f|^{s} d \bar{\mu}\right)^{1 / s}$. For $1 \leqq s, t<\infty$ and $p=\left\{p_{0}, p_{1}, \cdots, p_{m-1}\right\} L^{t}\left(M(\complement p) ; L^{s} M(p)\right)$ denotes the Lebesgue space with mixed norm

$$
\|f\|_{(t, 8: p)}=\left(\int_{M(\mathcal{C} p)}\left(\int_{M(p)}|f|^{8} d \mu(p)\right)^{t / 8} d \mu(\complement p)\right)^{1 / t} .
$$

The definition for $s=\infty$ or/and $t=\infty$ will be obvious.

Let $d=m+n$, where $m$ and $n$ are positive integers. Define $u$ by

$$
1 / u=(m / s+n / t) / d .
$$


For $1 \leqq s \leqq \infty, s^{\prime}$ will denote the conjugate exponent defined by $1 / s+$ $1 / s^{\prime}=1$.

For simple functions $w$ and $f$ in $(\bar{M}, \overline{\mathscr{G}}, \bar{\mu})$ we shall define holomorphic functions $W^{z}(x)$ and $F^{z}(x)$ and estimate them in mixed Lebesgue spaces. Our arguments are divided into two cases.

2.1. The case $m \geqq n$ and $\infty \geqq t \geqq s \geqq 1$. Let $P$ be the family of index sets $p$ of $\{0,1, \cdots, d-1\}$ with $\operatorname{card}(p)=m$. Let $Q=\{p \in P ; 0 \in p\}$ and $R=P-Q$. For $q \in Q$ put $R^{q}=\{r \in R ; \operatorname{card}(q \cap r)=m-n\}$. Then we have

$$
\operatorname{card}(P)=\left(\begin{array}{c}
d \\
m
\end{array}\right), \quad \operatorname{card}(Q)=\left(\begin{array}{c}
d-1 \\
m-1
\end{array}\right), \quad \operatorname{card}(R)=\left(\begin{array}{c}
d-1 \\
m
\end{array}\right)
$$

and

$$
\operatorname{card}\left(R^{q}\right)=\left(\begin{array}{c}
m-1 \\
n-1
\end{array}\right)
$$

Divide the unit circle $\partial \boldsymbol{D}$ into $\left(\begin{array}{l}d \\ m\end{array}\right)$ congruent $\operatorname{arcs} I_{p}, p \in P$.

Let $\alpha_{0}(z)$ and $\alpha_{q}(z), q \in Q$, be functions in the Hardy space $H^{2}(\boldsymbol{D})$ in the unit disk $\boldsymbol{D}$ having the following properties:

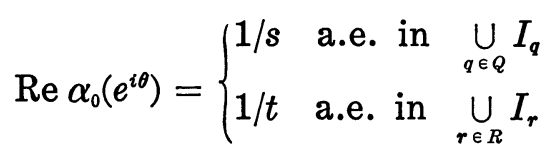

and

$$
\operatorname{Re} \alpha_{q}\left(e^{i \theta}\right)=\left\{\begin{array}{l}
1 / t-1 / s \text { a.e. in } I_{q} \\
(1 / s-1 / t) /\left(\operatorname{card}\left(R^{q}\right)\right) \text { a.e. in } \underset{r \in R^{q}}{\cup} I_{r} \\
0 \quad \text { a.e. in } \partial D-I_{q}-\bigcup_{r \in R^{q}} I_{r}
\end{array}\right.
$$

for each $q \in Q$. Furthermore we assume

$$
\operatorname{Im} \alpha_{0}(0)=\operatorname{Im} \alpha_{q}(0)=0 .
$$

By the mean value theorem we have

$$
\alpha_{0}(0)=[\operatorname{card}(Q) / s+\operatorname{card}(R) / t] \frac{1}{\operatorname{card}(P)}=(m / s+n / t) / d=1 / u
$$

and

$$
\alpha_{q}(0)=\frac{1 / t-1 / s}{\operatorname{card}(P)}+\frac{1 / s-1 / t}{\operatorname{card}\left(R^{q}\right)} \frac{\operatorname{card}\left(R^{q}\right)}{\operatorname{card}(P)}=0 .
$$

For a non zero function $w$ in $L^{u}(\bar{M})$ and $z \in \boldsymbol{D}$ define 


$$
W^{z}(x)=A_{w}(z) e^{i \arg w(x)}|w(x)|^{u \alpha_{0}(z)} \prod_{q \in Q}\left(\int_{M(q)}|w(x)|^{u} d \mu(q)\right)^{\alpha_{q}(z)}
$$

if $w(x) \neq 0$ and $=0$ otherwise, where

$$
A_{w}(z)=\|w\|_{u}^{u \gamma(z)}
$$

and

$$
\gamma(z)=\frac{m}{n}\left(\alpha_{0}(z)-\frac{1}{s}\right)+\left(\frac{1}{u}-\frac{1}{t}\right) .
$$

For a non zero function $f$ in $L^{u^{\prime}}(\bar{M})$ with $1 / u^{\prime}=1-1 / u$ and for $z \in \boldsymbol{D}$ define

$$
F^{z}(x)=B_{f}(z) e^{i \arg f(x)}|f(x)|^{u^{\prime}\left(1-\alpha_{0}(z)\right)} \prod_{q \in Q}\left(\int_{M(q)}|f(x)|^{u^{\prime}} d \mu(q)\right)^{-\alpha_{q}(z)}
$$

if $f(x) \neq 0$ and $=0$ otherwise, where

$$
B_{f}(z)=\|f\|_{u^{\prime}}^{u^{\prime} \gamma(z)} .
$$

LEMMA 1 . Let $d=m+n, m \geqq n \geqq 1$ and $\infty \geqq t \geqq s \geqq 1$. Let $w$ and $f$ be non zero functions $L^{u}(\bar{M})$ and $L^{u^{\prime}}(\bar{M})$ respectively. We have the followings.

(i) $\quad W^{0}(x)=w(x)$ and $F^{0}(x)=f(x)$.

(ii) Let $p \in P$ and $z \in \operatorname{int}\left(I_{p}\right)$. Then

$$
\left\|W^{z}\right\|_{(t, s: p)} \leqq\|w\|_{u} \text {. }
$$

(iii) Furthermore if $f$ is of the form $f_{0}\left(x_{0}\right) f_{1}\left(x_{1}\right) \cdots f_{d-1}\left(x_{d-1}\right)$,

$$
\left\|F^{z}\right\|_{\left(t^{\prime}, \varepsilon^{\prime} ; p\right)}=\|f\|_{u^{\prime}} \text {. }
$$

Proof. (i) follows easily from (2.1) and (2.2). To prove (ii) and (iii) we assume $1<s<t<\infty$. A proof for other cases is similar.

(ii) Assume $q \in Q$ and $z \in \operatorname{int}\left(I_{q}\right)$. Then

$$
\left|W^{z}(x)\right|^{s}=\left|A_{v o}(z)\right|^{s}|w(x)|^{u}\left(\int|w(x)|^{u} d \mu(q)\right)^{s / t-1}
$$

from which we get (ii).

Next suppose $r \in R$ and $z \in \operatorname{int}\left(I_{r}\right) . \quad \operatorname{Re} \alpha_{q}(z)=0$ for $q$ such that $r \notin R^{q}$ and $\operatorname{Re} \alpha_{q}(z)=v$ for $q$ such that $r \in R^{q}$, where

$$
v=(1 / s-1 / t) /\left(\begin{array}{c}
m-1 \\
n-1
\end{array}\right) \text {. }
$$

Therefore

$$
\left|W^{z}(x)\right|^{s}=\left|A_{v v}(z)\right|^{*}|w(x)|^{u s / t} \prod_{q \in S}\left(\int|w(x)|^{u} d \mu(q)\right)^{s v},
$$


where

$$
S=\left\{q \in Q ; r \in R^{q}\right\}
$$

Let $a \in r$ and put

$$
A=\{q \in S ; a \notin q\} \quad \text { and } B=\{q \in S ; a \in q\} .
$$

Then

$$
\operatorname{card}(S)=\left(\begin{array}{l}
m \\
n
\end{array}\right), \quad \operatorname{card}(A)=\left(\begin{array}{c}
m-1 \\
n-1
\end{array}\right)
$$

and

$$
\operatorname{card}(B)=\left(\begin{array}{c}
m-1 \\
n
\end{array}\right)
$$

if $m>n$ and $=0$ if $\mathrm{m}=n$.

Applying Hölder's inequality with exponents $(s / t)+s v \cdot \operatorname{card}(A)=1$ we have

$$
\begin{aligned}
& \int\left|W^{z}(x)\right|^{s} d \mu_{a} \\
& \quad=\left|A_{w}(z)\right|^{s} \prod_{q \in B}\left(\int|w(x)|^{u} d \mu(q)\right)^{s v} \int|w(x)|^{u s / t} \prod_{q \in A}\left(\int|w(x)|^{u} d \mu(q)\right)^{s v} d \mu_{a} \\
& \quad \leqq\left|A_{w}(z)\right|^{s} \prod_{q \in B}\left(\int|w(x)|^{u} d \mu(q)\right)^{s v} \prod_{q \in A}\left(\int|w(x)|^{u} d \mu(q) d \mu_{a}\right)^{s v}\left(\int|w(x)|^{u} d \mu_{a}\right)^{s / t} .
\end{aligned}
$$

Iterating this process for all $a$ in $r$ we get

$$
\int\left|W^{z}(x)\right|^{s} d \mu(r) \leqq\left|A_{w}(z)\right|^{s}\left(\int|w|^{u} d \mu(r)\right)^{s / t}\left(\int|w|^{u} d \bar{\mu}\right)^{s v}\left(\begin{array}{c}
m \\
n
\end{array}\right) .
$$

Since $s v\left(\begin{array}{c}m \\ n\end{array}\right)=(1-s / t) m / n$ and $\left|A_{w}(z)\right|=\|w\|_{u}^{1-u / t-u(1 / 8-1 / t) m / n}$, we have

$$
\int\left(\int\left|W^{z}\right|^{s} d \mu(r)\right)^{t / s} d \mu(\complement r) \leqq\left(\int|w|^{u} d \bar{\mu}\right)^{t / u},
$$

which proves (ii).

(iii) Let $f(x)=f_{0}\left(x_{0}\right) f_{1}\left(x_{1}\right) \cdots f_{d-1}\left(x_{d-1}\right)$ be a non zero function in $L^{u^{\prime}}(\bar{M})$. If $q \in Q$ and $z \in \operatorname{int}\left(I_{q}\right)$, then

$$
\left|F^{z}(x)\right|^{z^{\prime}}=\left|B_{f}(z)\right|^{z^{\prime}}|f(x)|^{u^{\prime}}\left(\int|f(x)|^{u^{\prime}} d \mu(q)\right)^{s^{\prime} / t^{\prime}-1} .
$$

Since $\left|B_{f}(z)\right|=\|f\|_{u}^{1-u^{\prime} / t^{\prime}}$,

$$
\int\left(\int\left|F^{z}\right|^{z^{\prime}} d \mu(q)\right)^{t^{\prime} / z^{\prime}} d \mu(\complement q)=\left|B_{f}(z)\right|^{t^{\prime}} \int|f(x)|^{u^{\prime}} d \bar{\mu}=\|f\|_{u^{\prime}}^{t^{\prime}} .
$$


Assume $r \in R$ and $z \in \operatorname{int}\left(I_{r}\right)$. Then

$$
\left|F^{z}(x)\right|^{\mathbf{s}^{\prime}}=\left.\left|B_{f}(z)\right|\right|^{s^{\prime}}|f(x)|^{u^{\prime} \mathbf{s}^{\prime} / t^{\prime}} \prod_{q \in \mathrm{s}}\left(\int|f(x)|^{u^{\prime}} d \mu(q)\right)^{-\mathbf{s}^{\prime} v},
$$

where $S$ is defined by (2.5). Let $r=\left\{r_{0}, r_{1}, \cdots, r_{m-1}\right\}$. Put $f(r)(x)=$ $f_{r_{0}}\left(x_{r_{0}}\right) f_{r_{1}}\left(x_{r_{1}}\right) \cdots f_{r_{m-1}}\left(x_{r_{m-1}}\right)$ and define $f(\complement r)$ for $\complement r=\{0,1, \cdots, d-1\}-r$ similarly. Suppose $a \in r$. Since $\{q \in S ; a \notin q\}=A$ by definition, the exponent of $f_{a}\left(x_{a}\right)$ in (2.9) equals $u^{\prime} s^{\prime} / t^{\prime}-u^{\prime} s^{\prime} v \operatorname{card}(A)=u^{\prime}$. On the other hand since $\{q \in S ; a \in q\}=B$, the exponent of $\left.\int\left|f_{a}\right|\right|^{\prime} d \mu_{a}$ is $-s^{\prime} v \operatorname{card}(B)=$ $\left(1-s^{\prime} / t^{\prime}\right)(m-n) / n$ by (2.8). Next consider the case $a \notin r$. We remark that $a \notin r$ implies that $a \in q$ for every $q \in S$. Thus the exponent of $f_{a}\left(x_{a}\right)$ in (2.9) is $u^{\prime} s^{\prime} / t^{\prime}$ and that of $\int\left|f_{a}\right|^{\prime} d \mu_{a}$ is $-s^{\prime} v \operatorname{card}(S)=\left(1-s^{\prime} / t^{\prime}\right) m / n$. Therefore we have

$$
\begin{aligned}
\left|F^{z}(x)\right|^{\mathbf{s}^{\prime}}= & \left|B_{f}(z)\right|^{\mathbf{s}^{\prime}}|f(r)(x)|^{u^{\prime}}\left(\int|f(r)|^{u^{\prime}} d \mu(r)\right)^{\left(1-\boldsymbol{s}^{\prime} / t^{\prime}\right)(m-n) / n} \\
& \times|f(\complement r)(x)|^{u^{\prime} \mathbf{s}^{\prime} / t^{\prime}}\left(\int|f(\complement r)|^{u^{\prime}} d \mu((\zeta r))^{\left(1-8^{\prime} / t^{\prime}\right) m / n} .\right.
\end{aligned}
$$

Thus

$$
\int\left(\int\left|F^{z}\right|^{\prime} d \mu(r)\right)^{t^{\prime} / \mathbf{s}^{\prime}} d \mu(\complement r)=\left|B_{f}(z)\right|^{t^{\prime}}\left(\int|f| u^{\prime} d \bar{\mu}\right)^{1+\left(t^{\prime} / \mathbf{s}^{\prime}-1\right) m / n} .
$$

Since $\left|B_{f}(z)\right|^{t^{\prime}}=\|f\|_{u^{\prime}}^{-t^{\prime} u^{\prime} \gamma(z)}=\|f\|_{u^{\prime}}^{-u^{\prime}+t^{\prime}-u^{\prime}\left(t^{\prime} / z^{\prime}-1\right) m / n}$, the right hand side of (2.10) equals $\|f\|_{u^{\prime}}^{t^{\prime}}$, which proves (iii).

2.2. The case $n>m$ and $\infty \geqq t \geqq s \geqq 1$. Let

$$
d=m k+r
$$

where $k \geqq 2$ and $m \geqq r>0$, so that $n=m(k-1)+r$.

We define a family $P$ of $m$ integers $p^{a}=\left\{p_{0}^{a}, p_{1}^{a}, \cdots p_{m-1}^{a}\right\}, 0 \leqq a<d$, as follows. If $0 \leqq j<k$ and $0 \leqq b<m-r$ or if $0 \leqq j<k-1$ and $m-r \leqq b<m$, then

$$
p_{c}^{m j+b} \equiv m j+b+c \quad \bmod d .
$$

If $j=k-1$ and $m-r \leqq b<m$ define

$$
\begin{aligned}
p^{m(k-1)+b}= & \{m(k-1)+b, m(k-1)+b+1, \cdots, m k-1\} \\
& \cup\{m(k-1)+b+r, m(k-1)+b+r+1, \cdots, m k+b-1\} \\
& \cup\{m(k+1), m(k+1)+1, \cdots, m(k+1)+b-m+r-1\} \\
& \bmod d .
\end{aligned}
$$

For $m k \leqq a<d$ put 


$$
p^{a}=\{m k, m k+1, \cdots, m k+m-1\} \bmod d .
$$

We remark the followings.

(2.11) For each $l=0,1, \cdots, m-1, p^{m j+l}(j=0,1, \cdots, k-1)$ are mutually disjoint and $\operatorname{card}\left(\cup_{j=0}^{k-1} p^{m j+l} \cup p^{a}\right)=d$ if $m k \leqq a<d$.

(2.12) If $a=m k+b \bmod d$ for some $b=0,1, \cdots, m-1$, then

$\operatorname{card}\left\{l ; a \notin p^{l} \cup p^{m+l} \cup \cdots \cup p^{m(k-1)+l}, 0 \leqq l<m\right\}=r$.

Let $\alpha_{0}(z)$ and $\alpha_{a}(z), m \leqq a<m(k+1)$, be functions in $H^{2}(\boldsymbol{D})$ which satisfy the following conditions: Divide the unit circle $\partial \boldsymbol{D}$ into $d$ congruent $\operatorname{arcs} I_{a}, a=0,1, \cdots, d-1$. We choose $\alpha_{0}$ and $\alpha_{a}$ so that

$$
\operatorname{Re} \alpha_{0}\left(e^{i \theta}\right)=\left\{\begin{array}{lll}
1 / s & \text { a.e. } & \text { in } \bigcup_{l=0}^{m-1} I_{l} \\
1 / t & \text { a.e. } & \text { in } \partial D-\bigcup_{l=0}^{m-1} I_{l},
\end{array}\right.
$$

for $1 \leqq j<k$ and $0 \leqq l<m$,

$$
\operatorname{Re} \alpha_{m j+l}\left(e^{i \theta}\right)=\left\{\begin{array}{lll}
1 / t-1 / s & \text { a.e. } & \text { in } I_{m(j-1)+l} \\
1 / s-1 / t & \text { a.e. } & \text { in } I_{m j+l} \\
0 & \text { a.e. } & \text { in } \partial D-I_{m(j-1)+l} \cup I_{m j+l}
\end{array}\right.
$$

and for $0 \leqq l<m$

$$
\operatorname{Re} \alpha_{m k+l}\left(e^{i \theta}\right)=\left\{\begin{array}{l}
1 / t-1 / s \text { a.e. in } I_{m(k-1)+l} \\
(1 / s-1 / t) / r \text { a.e. in } \bigcup_{b=0}^{r-1} I_{m k+b} \\
0 \text { a.e. in } \partial D-I_{m(k-1)+l} \cup \bigcup_{b=0}^{r-1} I_{m k+b} .
\end{array}\right.
$$

Furthermore we assmme that

$$
\operatorname{Im} \alpha_{0}(0)=\operatorname{Im} \alpha_{a}(0)=0 \text {. }
$$

Then we have

$$
\alpha_{0}(0)=1 / u \text { and } \alpha_{a}(0)=0 \text { for } m \leqq a<m(k+1) .
$$

Let $\gamma$ be a function in $H^{2}(\boldsymbol{D})$ such that $\operatorname{Re} \gamma\left(e^{i \theta}\right)=1 / u-1 / t$ a.e. in $\cup_{a=0}^{m k-1} I_{a}$ and $=(1 / u-1 / t)-(1 / s-1 / t) m / r$ a.e. in $\bigcup_{a=m k}^{d-1} I_{a}$, and $\operatorname{Im} \gamma(0)=$ 0 . Then we have $\gamma(0)=0$.

For a non zero function $w$ in $L^{u}(\bar{M})$ define

$$
\begin{aligned}
W^{z}(x)= & A_{w}(z)|w(x)|^{u \alpha_{0}(z)} e^{i \arg w(x)} \\
& \times \prod_{l=0}^{m-1} \prod_{j=1}^{k}\left(\int|w|^{u} d \mu\left(p^{l} \cup p^{m+l} \cup \cdots \cup p^{m(j-1)+l}\right)\right)^{\alpha_{m j}+l(z)}
\end{aligned}
$$


if $w(x) \neq 0$ and $=0$ otherwise, where $A_{w}(z)=\|w\|_{u}^{u \gamma(z)}$. For a non zero function $f$ in $L^{u^{\prime}}(\bar{M})$ put

$$
\begin{aligned}
F^{z}(x)= & B_{f}(z)|f(x)|^{u^{\prime}\left(1-\alpha_{0}(z)\right)} e^{i \arg f(x)} \\
& \times \prod_{l=0}^{m-1} \prod_{j=1}^{k}\left(\int|f|^{u^{\prime}} d \mu\left(p^{l} \cup p^{m+l} \cup \cdots \cup p^{m(j-1)+l}\right)\right)^{-\alpha_{m j+l}(z)}
\end{aligned}
$$

if $f(x) \neq 0$ and $=0$ otherwise, where $B_{f}(z)=\|f\|_{u^{\prime}}^{u^{\prime} \gamma(z)}$.

LEMMA 2. Let $d=m+n, n>m \geqq 1$ and $\infty \geqq t \geqq s \geqq 1$. Let $w$ and $f$ be non zero functions in $L^{u}(\bar{M})$ and $L^{u^{\prime}}(\bar{M})$ respectively. We have the followings.

$$
W^{0}(x)=w(x) \text { and } F^{0}(x)=f(x) .
$$

(ii) Let $0 \leqq a<d$ and $z \in \operatorname{int}\left(I_{a}\right)$. Then

$$
\left\|W^{z}\right\|_{\left(t, s: p^{a}\right)} \leqq\|w\|_{u} \text {. }
$$

(iii) Furthermore if $f$ is of the form $f_{0}\left(x_{0}\right) f_{1}\left(x_{1}\right) \cdots f_{d-1}\left(x_{d-1}\right)$,

$$
\left\|F^{z}\right\|_{\left(t^{\prime}, s^{\prime}: p^{a}\right)}=\|f\|_{u^{\prime}} \text {. }
$$

Proof. (i) is obvious. To prove (ii) and (iii) we assume $1<s<t<$ $\infty$. The other case is proved similarly.

(ii) First suppose that $0 \leqq b<m$ and $z \in \operatorname{int}\left(I_{b}\right)$. If $m j+b \neq 0$ and if $\alpha_{m j+l}(z) \neq 0$ then $j=1, l=b$ and $\alpha_{m+b}(z)=1 / t-1 / s$. Thus

$$
\left|W^{z}(x)\right|^{s}=\left|A_{w}(z)\right|^{s}|w(x)|^{u}\left(\int|w|^{u} d \mu\left(p^{b}\right)\right)^{s / t-1} .
$$

Since $\left|A_{w}(z)\right|=\|w\|_{u}^{1-u / t}$,

$$
\int\left(\int\left|W^{z}\right|^{s} d \mu\left(p^{b}\right)\right)^{t / s} d \mu\left(\complement p^{b}\right)=\left|A_{w}(z)\right|^{t} \int|w|^{u} d \mu\left(p^{b}\right) d \mu\left(\complement p^{b}\right)=\left(\int|w|^{u} d \bar{\mu}\right)^{t / u} .
$$

Next suppose that $0 \leqq b<m, 1 \leqq j<k$ and $z \in \operatorname{int}\left(I_{m j+b}\right)$. Remark that $\operatorname{Re} \alpha_{0}(z)=1 / t, \operatorname{Re} \alpha_{m(j+1)+b}(z)=1 / t-1 / s, \quad \operatorname{Re} \alpha_{m j+b}(z)=1 / s-1 / t$ and $\operatorname{Re} \alpha_{a}(z)=0$ for $a \neq 0, m j+b, m(j+1)+b$. Thus

$$
\begin{aligned}
\left|W^{z}(x)\right|^{s}= & \left|A_{w}(z)\right|^{s}|w(x)|^{u z / t}\left(\int|w|^{u} d \mu\left(p^{b} \cup p^{m+b} \cup \cdots \cup p^{m(j-1)+b}\right)\right)^{1-s / t} \\
& \times\left(\int|w|^{u} d \mu\left(p^{b} \cup p^{m+b} \cup \cdots \cup p^{m j+b}\right)\right)^{s / t-1} .
\end{aligned}
$$

Integrate both sides with respect to $d \mu\left(p^{m j+b}\right)$ and apply Hölder's inequality with exponents $s / t+(1-s / t)=1$. Then we get

$$
\int\left|W^{z}\right|^{s} d \mu\left(p^{m j+b}\right) \leqq\left|A_{w}(z)\right|^{s}\left(\int|w|^{u} d \mu\left(p^{m j+b}\right)\right)^{s / t},
$$


which proves (ii) by the same way as above.

Finally suppose that $0 \leqq b<r$ and $z \in \operatorname{int}\left(I_{m k+b}\right)$. By definition

$$
\begin{aligned}
\left|W^{z}(x)\right|^{s}= & \left|A_{w}(z)\right|^{s}|w(x)|^{u s / t} \\
& \times \prod_{l=0}^{m-1}\left(\int|w|^{u} d \mu\left(p^{l} \cup p^{m+l} \cup \cdots \cup p^{m(k-1)+l}\right)\right)^{(1-8 / t) / r} .
\end{aligned}
$$

Recall that $p^{m k+b}=\{m k, m k+1, \cdots, m k+m-1\} \bmod d$. Let $a \in p^{m k+b}$ and set $A=\left\{l ; a \notin p^{l} \cup p^{m+l} \cup \cdots \cup p^{m(k-1)+l}, 0 \leqq l<m\right\}$ and $B=\{l \notin A ; 0 \leqq$ $l<m\}$. Then by (2.12) card $(A)=r$. Furthermore we have $p^{l} \cup^{m+l} \cup$ $\cdots \cup p^{m(k-1)+l} \cup p^{m k+b}=\{0,1, \cdots, d-1\}$. Applying Hölder's inequality to (2.15) with exponents $s / t+r \cdot(1-s / t) / r=1$, we have

$$
\begin{aligned}
\int\left|W^{z}\right|^{s} d \mu_{a} \leqq & \left|A_{w}(z)\right|^{s}\left(\int|w|^{u} d \mu_{a}\right)^{s / t} \\
& \times \prod_{l \in A}\left(\int|w|^{u} d \mu\left(p^{l} \cup p^{m+l} \cup \cdots \cup p^{m(k-1)+l} \cup\{a\}\right)\right)^{(1-s / t) / r} \\
& \times \prod_{l \in B}\left(\int|w|^{u} d \mu\left(p^{l} \cup p^{m+l} \cup \cdots \cup p^{m(k-1)+l}\right)\right)^{(1-s / t) / r} .
\end{aligned}
$$

Iterating this process for all $a \in p^{m k+b}$ and using (2.11), we get finally

$$
\int\left|W^{z}\right|^{s} d \mu\left(p^{m k+b}\right) \leqq\left|A_{w}(z)\right|^{s}\left(\int|w|^{u} d \mu\left(p^{m k+b}\right)\right)^{s / t}\left(\int|w|^{u} d \bar{\mu}\right)^{(1-s / t) m / r} .
$$

Integrating both sides with respect to $d \mu\left(\complement p^{m k+b}\right)$ we get

$$
\int\left(\int\left|W^{z}\right|^{s} d \mu\left(p^{m k+b}\right)\right)^{t / s} d \mu\left(\complement p^{m k+b}\right) \leqq\left(\int|w|^{u} d \bar{\mu}\right)^{t / s},
$$

since $\left|A_{w}(z)\right|=\|w\|_{u}^{(1-u / t)-u(1 / s-1 / t) m / r}$, which proves (ii).

To prove the equality (iii) we consider only the case $z \in \operatorname{int}\left(I_{m k+b}\right)$ with $0 \leqq b<r$. A proof for other cases follows from similar arguments to (ii). By the definition (2.14)

$$
\begin{aligned}
\left|F^{z}(x)\right|^{s^{\prime}}= & \left|B_{f}(z)\right|^{s^{\prime}}|f(x)|^{u^{\prime} s^{\prime} / t^{\prime}} \\
& \times \prod_{l=0}^{m-1}\left(\int|f|^{u^{\prime}} d \mu\left(p^{l} \cup p^{l+m} \cup \cdots \cup p^{m(k-1)+l}\right)\right)^{\left(1-s^{\prime} / t^{\prime}\right) / r} .
\end{aligned}
$$

Assume $a \in p^{m k+b}$ and $A$ is the set defined in (ii). Since card $(A)=r$ the exponent of $f_{a}\left(x_{a}\right)$ in $\left|F^{z}\right|^{z^{\prime}}$ is $u^{\prime} s^{\prime} / t^{\prime}+r \cdot u^{\prime}\left(1-s^{\prime} / t^{\prime}\right) / r=u^{\prime}$ and the one of $\int\left|f_{a}\right|^{\prime} d \mu_{a}$ equals $(m-r)\left(1-s^{\prime} / t^{\prime}\right) / r$. If $a \notin p^{m k+b}$ the term containing $f_{a}$ in $\left|F^{2}(x)\right|^{\prime \prime}$ is

$$
\left|f_{a}\left(x_{a}\right)\right|^{u^{\prime} s^{\prime} / t^{\prime}}\left(\int\left|f_{a}\right|^{u^{\prime}} d \mu_{a}\right)^{m\left(1-s^{\prime} / t^{\prime}\right) / r}
$$


Thus

$$
\begin{aligned}
\int\left|F^{z}\right|^{s^{\prime}} d \mu\left(p^{m k+b}\right)= & \left|B_{f}(z)\right|^{s^{\prime}}\left|f\left(\mathfrak{C} p^{m k+b}\right)(x)\right|^{u^{\prime} s^{\prime} / t^{\prime}} \\
& \times\left(\int\left|f\left(p^{m k+b}\right)\right|^{u^{\prime}} d \mu\left(p^{m k+b}\right)\right)^{1+(m-r)\left(1-s^{\prime} / t^{\prime}\right) / r} \\
& \times\left(\int \mid f\left(\left.\complement\left(p^{m k+b}\right)\right|^{u^{\prime}} d \mu\left(\mathfrak{C} p^{m k+b}\right)\right)^{m\left(1-s^{\prime} / t^{\prime}\right) r}\right.
\end{aligned}
$$

Since $\left|B_{f}(z)\right|=\|f\|_{u^{\prime}}^{u^{\prime}\left[\left(1 / u^{\prime}-1 / t^{\prime}\right)-\left(1 / \mathbf{s}^{\prime}-1 / t^{\prime}\right) m / r\right]}$, we get

$$
\left.\int\left(\int\left|F^{z}\right|^{s^{\prime}} d \mu\left(p^{m k+b}\right)\right)\right)^{t^{\prime} / s^{\prime}} d \mu\left(\complement p^{m k+b}\right)=\left(\int|f|^{u^{\prime}} d \bar{\mu}\right)^{t^{\prime} / u^{\prime}}
$$

which proves (iii).

REMARK 1. If $d=m k$ for some $k \geqq 3$, then Lemma 2.2 (iii) holds for functions $f$ of the form

$$
f_{0}\left(x_{0}, \cdots, x_{m-1}\right) f_{1}\left(x_{m}, \cdots x_{2 m-1}\right) \cdots f_{k-1}\left(x_{m(k-1)}, \cdots, x_{m k-1}\right) \text {. }
$$

3. Interpolation theorems. Let $d=m+n$. Let $P$ be the family of $m$ integers defined in 2.1 or 2.2 according to $m \geqq n$ or $m<n$ and $I_{p}$, $p \in P$, be arcs in $\S 2$. Let $(M, \mathscr{N}, \mu)$ and $(N, \mathscr{N}, \nu)$ be $\sigma$-finite measure spaces. $(M(p), \mathscr{M}(p), \mu(p)),(\bar{M}, \overline{\mathscr{H}}, \bar{\mu}),(N(p), \mathscr{N}(p), \nu(p))$ and $(\bar{N}, \overline{\mathscr{N}}, \bar{\nu})$ will denote the product spaces defined in $\S 2$.

THEOREM 1. Let $T$ be a linear operator of simple functions on $(\bar{M}, \overline{\mathscr{L}}, \bar{\mu})$ to measurable functions on $(N, \mathscr{N}, \nu)$. Let $v\left(e^{i \theta}\right)$ be a measurable function in $\partial \boldsymbol{D}$ such that $1 \leqq v\left(e^{i \theta}\right) \leqq \infty$. Define $v$ by

$$
1 / v=\int_{\partial \boldsymbol{D}}\left(1 / v\left(e^{i \theta}\right)\right) \frac{d \theta}{2 \pi}
$$

Let $1 \leqq u_{0} \leqq u_{1} \leqq \infty$ and

$$
1 / u=\left(m / u_{0}+n / n_{1}\right) / d
$$

Suppose that

$$
\|T w\|_{v\left(e^{i \theta}\right)} \leqq C\left(e^{i \theta}\right)\|w\|_{\left(u_{1}, u_{0}: p\right)}
$$

for simple functions $w$ and $e^{i \theta} \in \operatorname{int}\left(I_{p}\right), p \in P$, where $C\left(e^{i \theta}\right)$ is measurable on $\partial D$. Then

$$
\|T w\|_{v} \leqq C\|w\|_{u},
$$

where

$$
C=\exp \int_{\partial \boldsymbol{D}} \log C\left(e^{i \theta}\right) \frac{d \theta}{2 \pi}
$$


REMARK 1. If $1 \leqq u_{1} \leqq u_{0} \leqq \infty$, (3.3) holds for $w$ of the form $w_{0}\left(x_{0}\right) w_{1}\left(x_{1}\right) \cdots w_{d-1}\left(x_{d-1}\right)$ under the assumption (3.2) with $w$ as above. This is a consequence of Lemma 2 (see also [1]), but in general we cannot conclude (3.3) for all $w$ in $L^{u}(\bar{M})$. We shall give a counter example in $\S 5$, Remark 4 .

REMARK 2. The family of the spaces $L^{v\left(e^{i \theta}\right)}(N)$ in Theorem 1 is replaced by more general family of Banach spaces $B[z], z \in \partial D$, which is introduced by Coifman, Cwikel, Rochberg, Sagher and Weiss [3].

Proof. Let $P_{z}\left(e^{i \theta}\right), z=r e^{i \tau} \in \boldsymbol{D}$, be the Poisson kernel $\left(1-r^{2}\right) /(1-2 r$ $\left.\cos (\theta-\tau)+r^{2}\right)$. Denote by $v^{\prime}\left(e^{i \theta}\right)$ the conjugate exponent of $v\left(e^{i \theta}\right)$. Let $V^{\prime}(z)$ be a holomorphic function in $\boldsymbol{D}$ such that

$$
\operatorname{Re} V^{\prime}\left(e^{i \theta}\right)=1 / v^{\prime}\left(e^{i \theta}\right)=1-1 / v\left(e^{i \theta}\right) \text { a.e. }
$$

and

$$
\operatorname{Im} V^{\prime}(0)=0 .
$$

Such a function exists in the space $H^{2}(\boldsymbol{D})$ and $V^{\prime}(0)=1-1 / v=1 / v^{\prime}$.

Suppose $w$ and $g$ are non null simple functions in $N$ and $\|g\|_{v^{\prime}}=1$. Define $W^{z}$ by (2.3) or (2.13) and $G^{z}=e^{\arg g(x)}|g|^{v^{\prime} V^{\prime}(z)}$. Put

$$
\Phi(z)=\int_{N} T W^{z} \cdot G^{z} d \nu .
$$

$\Phi(z)$ belongs to the class $N_{+}(\boldsymbol{D})$, which consists of holomorphic functions $\phi$ in $\boldsymbol{D}$ such that $\sup _{0<r<1} \int_{\partial \boldsymbol{D}} \log ^{+}\left|\phi\left(r e^{i \theta}\right)\right| d \theta<\infty$ and

$$
\log |\phi(z)| \leqq \int_{\partial \boldsymbol{D}} \log \left|\phi\left(e^{i \theta}\right)\right| P_{z}\left(e^{i \theta}\right) \frac{d \theta}{2 \pi}
$$

for $z \in \boldsymbol{D}$ where $\log ^{+} x=\max (0, \log x)$ (see [3]).

Let $p \in P$ and assume $z=e^{i \theta} \in \operatorname{int}\left(I_{p}\right)$. Then by Hölder's inequality and (3.2)

$$
|\Phi(z)| \leqq\left\|T W^{z}\right\|_{v(z)}\left\|G^{z}\right\|_{v^{\prime}(z)} \leqq C(z)\left\|W^{z}\right\|_{\left(u_{0}, u_{1}: p\right)}\left\|G^{z}\right\|_{v^{\prime}(z)} .
$$

By Lemma 1 or 2 we have $\left\|W^{2}\right\|_{\left(u_{1}, u_{0}: p\right)} \leqq\|w\|_{u}$. Furthermore we have $\left\|G^{z}\right\|_{v^{\prime}(z)}=\|g\|_{v^{\prime} / v^{\prime}(z)}^{v^{\prime}}=1$. Thus

$$
|\Phi(z)| \leqq C(z)\|w\|_{u} \text {. }
$$

Applying Jensen's inequality (3.4) to $\Phi$ and $z=0$ we get

$$
|\Phi(0)| \leqq \exp \int_{\partial \boldsymbol{D}} \log C\left(e^{i \theta}\right) \frac{d \theta}{2 \pi}\|w\|_{u} .
$$

Taking supremum over $g$ such that $\|g\|_{v^{\prime}}=1$ we get (3.3). 
We can generalize Theorem 1 for analytic family of operators $\left\{T^{z}\right\}$, $z \in D . \quad\left\{T^{2}\right\}$ is said to be an analytic family if

$$
\Phi(z)=\int_{N} T^{z} W^{z} \cdot G^{z} d \nu
$$

belongs to $N_{+}(\boldsymbol{D})$ for every simple function $w$ and $g$ on $N$.

THEOREM 1'. Let $\left\{T^{z}\right\}$ be an analytic family of operators. Under the assumption of Theorem 1 if

$$
\left\|T^{z} w\right\|_{v(z)} \leqq C(z)\|w\|_{\left(u_{1}, u_{0}: p\right)}
$$

for $z=e^{i \theta} \in \operatorname{int}\left(I_{p}\right), p \in P$, then we have

$$
\left\|T^{0} w\right\|_{v} \leqq C\|w\|_{u} .
$$

THEOREM 2. Let $T$ be a linear operator of simple functions on $\bar{M}$ to measurable functions on $\bar{N}$. Let $1 \leqq u_{0} \leqq u_{1} \leqq \infty$ and $1 \leqq v_{1} \leqq v_{0} \leqq \infty$. Suppose that

$$
\|T w\|_{\left(v_{1}, v_{0}: p\right)} \leqq C(p)\|w\|_{\left(u_{1}, u_{0}: p\right)}
$$

for all $w$ and $p \in P$.

If

$$
1 / u=\left(m / u_{0}+n / u_{1}\right) / d \quad \text { and } 1 / v=\left(m / v_{0}+n / v_{1}\right) / d
$$

then

$$
\|T w\|_{v} \leqq C\|w\|_{u}
$$

where $C=\left(\prod_{p \in P} C(p)\right)^{1 / \operatorname{card}(P)}$.

Proof. Let $w$ and $g$ be non null simple functions on $\bar{M}$ and $\bar{N}$ respectively. Define $W^{z}$ and $G^{z}$ by $(2.3)$ with respect to indices $\left(u_{0}, u_{1}\right)$ and $\left(v_{0}^{\prime}, v_{1}^{\prime}\right)$ respectively. Put

$$
\Phi(z)=\int_{\bar{N}} T W^{z} \cdot G^{z} d \bar{\nu} .
$$

Obviously $\Phi(z) \in N_{+}(\boldsymbol{D})$. If $p \in P$ and $z \in \operatorname{int}\left(I_{p}\right)$, by Hölder's inequality and our assumption

$$
|\Phi(z)| \leqq\left\|T W^{z}\right\|_{\left(v_{1}, v_{0}: p\right)}\left\|G^{z}\right\|_{\left(v_{1}^{\prime}, v_{0}^{\prime} p p\right)} \leqq C(p)\left\|W^{z}\right\|_{\left(u_{1}, u_{0}: p\right)}\left\|G^{z}\right\|_{\left(v_{1}^{\prime}, v_{0}^{\prime}: p\right)} .
$$

Since $1 \leqq v_{0}^{\prime} \leqq v_{1}^{\prime} \leqq \infty$, the last two terms are estimated by Lemma 1 or 2 and consequently the last term is bounded by $C(p)\|w\|_{u}\|g\|_{v^{\prime}}$. By Jensen's inequality (3.4) we get

$$
|\Phi(0)| \leqq \exp \left(\sum_{p} \int_{I_{p}} \log C(p) \frac{d \theta}{2 \pi}\right)\|w\|_{u}\|g\|_{v^{\prime}},
$$

which proves our theorem, 
THEOREM 3. Let $T$ be a linear operator of simple functions on $\bar{M}$ of the form $f_{0}\left(x_{0}\right) f_{1}\left(x_{1}\right) \cdots f_{d-1}\left(x_{d-1}\right)$ to measurable functions on $\bar{N}$. Let $1 \leqq$ $u_{1} \leqq u_{0} \leqq \infty$ and $1 \leqq v_{1} \leqq v_{0} \leqq \infty$. Suppose that

$$
\|T f\|_{\left(v_{1}, v_{0}: p\right)} \leqq C(p)\|f\|_{\left(u_{1}, u_{0}: p\right)}
$$

for all simple function $f$ on the above form and $p \in P$.

If $u$ and $v$ are defined by (3.5), then

$$
\|T f\|_{v} \leqq C\|f\|_{u}
$$

for all $f$ of product form, where $C=\prod_{p \in P} C(p)^{1 / \operatorname{ard}(P)}$.

Proof. Assume $m \geqq n$. For simple non null functions $w(x)$ on $\bar{M}$ and $f(x)$ on $\bar{N}$ of product form define $W^{z}$ and $F^{z}$ by (2.3) and (2.4) with $\left(v_{0}^{\prime}, v_{1}^{\prime}\right)$ and $\left(u_{0}, u_{1}\right)$ respectively. Remark that $F^{z}$ is of product form too. Put $\Phi(z)=\int_{\bar{N}} T F^{z} \cdot W^{z} d \bar{\nu}$. If $p \in P$ and $z \in \operatorname{int}\left(I_{p}\right)$,

$$
|\Phi(z)| \leqq\left\|T F^{z}\right\|_{\left(v_{1}, v_{0}: p\right)}\left\|W^{z}\right\|_{\left(v_{1}^{\prime}, v_{0}^{\prime}: q\right)} \leqq C(p)\left\|F^{z}\right\|_{\left(u_{1}, u_{0}: p\right)}\left\|W^{z}\right\|_{\left(v_{1}^{\prime}, v_{0}^{\prime}: p\right)} .
$$

Since $1 \leqq v_{0}^{\prime} \leqq v_{1}^{\prime} \leqq \infty$, Lemma 1 is applied to the last term and we get our theorem by the same method as in the proof of Theorem 2 .

A proof for the case $m<n$ proceeds similarly applying Lemma 2 .

\section{Part II. Applications to Fourier analysis.}

4. Riesz-Bochner operator. Our aim in this section is to show Theorem 6 in $\S 4.4$. The idea of the proof is to estimate $s^{\varepsilon}(f)$ in the mixed Lebesgue space $L^{4}\left(\boldsymbol{R}^{2} ; L^{2}\left(\boldsymbol{R}^{d-2}\right)\right)$ applying the two dimensional argument due to Cordoba [4] and to use our interpolation theorem.

4.1. We introduce the operator $s$ as follows. Let $\phi$ be a $C^{\infty}$-function on the real line such that supp $\phi \subset(-1,1)$ and $\phi \geqq 0$. Fix $0<\delta<1 / 4$. For a function $f$ in $\mathscr{S}\left(\boldsymbol{R}^{d}\right)$ define $s(f)$ by the Fourier transform;

$$
s(f)^{\wedge}(\xi)=\phi\left(\left(1-|\xi|^{2}\right) \delta^{-1}\right) \hat{f}(\xi) .
$$

Now we shall consider a decomposition of $s(f)$. In the following $p$ denotes the set $\{0,1, \cdots, d-3\}$ and use the notations $\bar{x}=x(p)=\left(x_{0}, x_{1}\right.$. $\left.\cdots, x_{d-3}\right)$ and $\overline{\bar{x}}=x(\mathfrak{C} p)=\left(x_{d-2}, x_{d-1}\right)$ for $x \in \boldsymbol{R}^{d}$. Let $\psi$ be a $C^{\infty}$-function on the real line such that supp $\psi \subset(-5,5), \psi \geqq 0$ and $\psi=1$ on $(-4,4)$. For a positive integer $k$ put $\rho_{k}=1-\delta k$ if $0<\delta k \leqq(2-\sqrt{2}) / 2$ and $\left(1-(\sqrt{2}-1+\delta k)^{2}\right)^{1 / 2}$ if $(2-\sqrt{2}) / 2<\delta k<2-\sqrt{2}$. Let $\xi^{k, b}(\mathcal{C} p)=$ $\rho_{k}\left(\cos \delta^{1 / 2} \rho_{k}^{-1} b, \sin \delta^{1 / 2} \rho_{k}^{-1} b\right)$ for $b=0,1, \cdots$ and put

$$
\psi_{k, b}(\xi)=\psi\left(\left|\xi(\mathfrak{C} p)-\xi^{k, b}(\mathfrak{C} p)\right| \delta^{-1 / 2}\right) \psi\left(\left(|\xi(\mathfrak{C} p)|^{2}-\rho_{k}^{2}\right) \delta^{-1}\right) .
$$

If $\xi \in \operatorname{supp} \phi\left(\left(1-|\cdot|^{2}\right) \delta^{-1}\right)$, then $\psi_{k, b}(\xi) \neq 0$ for some $k$ and $b$ and the 
number of such $(k, b)$ 's is uniformly bounded. If we put $\Psi_{k, b}(\xi)=$ $\psi_{k, b}(\xi) / \sum_{l, j} \psi_{l, j}(\xi)$ where the denominator does not vanish, then $\left\{\Psi_{k, b}\right\}$ is a partition of unity of the support of $\phi\left(\left(1-|\cdot|^{2}\right) \delta^{-1}\right)$.

Let $\left\{\xi^{a}(p)\right\}$ be a sequence in $\boldsymbol{R}^{d-2}$ such that

$$
\delta=\inf _{i \neq j}\left|\xi^{i}(p)-\xi^{j}(p)\right| .
$$

Put

$$
\psi^{a}(\xi)=\psi\left(\left|\xi(p)-\xi^{a}(p)\right| \delta^{-1}\right)
$$

and $\Psi^{a}(\xi)=\psi^{a}(\xi) / \sum_{i} \psi^{i}(\xi)$.

Let us define $s_{k, b}^{a}(f)$ by

$$
s_{k, b}^{a}(f)^{\wedge}(\xi)=\phi\left(\left(1-|\xi|^{2}\right) \delta^{-1}\right) \Psi^{a}(\xi) \Psi_{k, b}(\xi) \hat{f}(\xi) .
$$

Furthermore put

$$
s_{k, b}(f)=\sum_{a} s_{k, b}^{a}(f) \quad \text { and } \quad s^{a}(f)=\sum_{k, b} s_{k, b}^{a}(f) .
$$

We get a decomposition of $s(f)$;

$$
s(f)=\sum_{k, b} \sum_{a} s_{k, b}^{a}(f) .
$$

In the following we denote the Fourier transform with respect to $x(p)$ and $x(\mathfrak{C} p)$ by $\mathscr{F}_{p}$ and $\mathscr{F}_{\mathfrak{c}_{p}}$ respectively. Thus $\hat{f}=\mathscr{F}_{p} \mathscr{F}_{\mathfrak{c}_{p}} f$.

LEMMA 3. We have

$$
\int_{R^{2}}\left(\int_{R^{d-2}}|s(f)|^{2} d x(p)\right)^{2} d x(\complement p) \leqq C \int_{R^{2}}\left(\int_{R^{d-2}} \sum_{a} \sum_{k, b}\left|s_{k, b}^{a}(f)\right|^{2} d x(p)\right)^{2} d x(\complement p p)
$$

for $f$ in $\mathscr{S}\left(\boldsymbol{R}^{d}\right)$, where the constant $C$ is independent of $f$ and $\delta$.

ProoF. Since $\mathscr{F}_{p} s_{k, b}^{a}(f)=\Psi^{a} \mathscr{F}_{p} s_{k, b}(f)$ and since supp $\Psi^{a}(a=1,2, \cdots)$ intersect at most $10^{d-2}$ times,

$$
\int_{\boldsymbol{R}^{d-2}}\left|s_{k, b}(f)(\bar{x}, \overline{\bar{x}})\right|^{2} d \bar{x} \leqq 10^{d-2} \sum_{a} \int_{\boldsymbol{R}^{d-2}}\left|s_{k, b}^{a}(f)(\bar{x}, \overline{\bar{x}})\right|^{2} d \bar{x}
$$

for all $\overline{\bar{x}}$. Therefore it suffices to show that

$$
\int_{R^{2}}\left(\int_{R^{d-2}}|s(f)|^{2} d \bar{x}\right)^{2} d \overline{\bar{x}} \leqq C \int_{R^{2}}\left(\int_{R^{d-2}} \sum_{k, b}\left|s_{k, b}(f)\right|^{2} d \bar{x}\right)^{2} d \overline{\bar{x}} .
$$

Dividing the sum $\sum_{k, b} s_{k, b}(f)$ into 100 sums we may assume $s(f)=$ $\sum s_{100 k, b}(f)$. If $\Psi_{100 k, a}(\bar{\xi}, \bar{\xi}) \phi\left(\left(1-|(\bar{\xi}, \bar{\xi})|^{2}\right) \delta^{-1}\right) \neq 0$ for some $\bar{\xi}$, then $\Psi_{100 j, b}(\bar{\xi}, \overline{\bar{\eta}}) \phi\left(\left(1-|(\bar{\xi}, \overline{\bar{\eta}})|^{2}\right) \delta^{-1}\right)=0$ for any $j \neq k, b$ and $\overline{\bar{\eta}}$. Thus the support of $\mathscr{F}_{p}\left(\sum_{b} s_{100 k, b}(f)\right)(\cdot, \overline{\bar{x}}), k=1,2, \cdots$, are disjoint for each $\overline{\bar{x}}$ in $\boldsymbol{R}^{2}$. In order to prove (4.2) we may assume $s(f)=\sum s_{100 k, 1006}(f)$ where 
$b<\delta^{-1 / 2} \rho_{k} / 200$. We denote simply $s_{k, b}(f)$ for $s_{100 k, 100 b}(f)$.

By Parseval relation we have

$$
\begin{aligned}
\int_{R^{d-2}}|s(f)(\bar{x}, \overline{\bar{x}})|^{2} d \bar{x} & =\int_{R^{d-2}}\left|\sum_{k} \mathscr{F}_{p}\left(\sum_{b} s_{k, b}(f)\right)(\bar{\xi}, \overline{\bar{x}})\right|^{2} d \bar{\xi} \\
& =\sum_{k} \int_{R^{d-2}}\left|\mathscr{F}_{p}\left(\sum_{b} s_{k, b}(f)\right)(\bar{\xi}, \overline{\bar{x}})\right|^{2} d \bar{\xi} .
\end{aligned}
$$

Thus

$$
\begin{aligned}
& \int_{R^{2}}\left(\int_{R^{d-2}}|s(f)(x)|^{2} d \bar{x}\right)^{2} d \overline{\bar{x}} \\
& \quad=\left.\sum_{k, l} \int_{R^{2}} d \overline{\bar{x}} \int_{R^{d-2}}\left|\mathscr{F}_{p}\left(\sum_{b} s_{k, b}(f)\right)(\bar{\xi}, \overline{\bar{x}})\right|^{2} d \bar{\xi} \int_{R^{d-2}} \mathscr{F}_{p}\left(\sum_{c} s_{l, c}(f)\right)(\bar{\eta}, \overline{\bar{x}})\right|^{2} d \bar{\eta} .
\end{aligned}
$$

Put

$$
\sigma_{(k, b i l, c)}(\bar{\xi}, \bar{\eta}, \overline{\bar{\xi}})=\frac{1}{2 \pi} \int_{R^{2}} \hat{s}_{k, b}(f)(\bar{\xi}, \overline{\bar{\xi}}-\overline{\bar{\eta}}) \hat{s}_{l, c}(f)(\bar{\eta}, \overline{\bar{\eta}}) d \bar{\eta} .
$$

Then the last sum equals, by convolution relation,

$$
\sum_{k} \sum_{b, b^{\prime}} \sum_{l} \sum_{c, c^{\prime}} \int d \bar{\xi} d \bar{\eta} \int \sigma_{(k, b ; l, c)}(\bar{\xi}, \bar{\eta}, \overline{\bar{\xi}}) \overline{\sigma_{\left(k, b^{\prime}: l, c^{\prime}\right)}(\bar{\xi}, \bar{\eta}, \overline{\bar{\xi}})} d \overline{\bar{\xi}} \text {. }
$$

Sublemma. If $(b, c) \neq\left(b^{\prime}, c^{\prime}\right)$, then

$$
\left.\sigma_{(k, b ; l, c)}(\bar{\xi}, \bar{\eta}, \bar{\xi}) \cdot \sigma_{\left(k, b^{\prime}: l, c^{\prime}\right)}(\bar{\xi}, \bar{\eta}, \overline{\bar{\xi}})\right) \equiv 0 \text {. }
$$

Granting for a moment this sublemma we have

$$
\int\left(\int|s(f)|^{2} d \bar{x}\right)^{2} d \overline{\bar{x}}=\sum_{k, b} \sum_{l, c} \int d \bar{\xi} d \bar{\eta} \int\left|\sigma_{(k, b ; l, c)}(\bar{\xi}, \bar{\eta}, \bar{\xi})\right|^{2} d \overline{\bar{\xi}},
$$

which is carried by Fourier transform with respect to $\bar{\xi}$ and then $\bar{\xi}, \bar{\eta}$ to the sum

$$
\begin{aligned}
\sum_{k, b} \sum_{l, c} \int d \overline{\bar{x}} \int\left|\mathscr{F}_{p} s_{k, b}(f)(\bar{\xi}, \overline{\bar{x}}) \mathscr{F}_{p} s_{l, c}(f)(\bar{\eta}, \overline{\bar{x}})\right|^{2} d \bar{\xi} d \bar{\eta} \\
=\sum_{k, b} \sum_{l, c} \int d \overline{\bar{x}}\left(\int\left|s_{k, b}(f)(x)\right|^{2} d \bar{x}\right)\left(\int\left|s_{l, c}(f)(x)\right|^{2} d \bar{x}\right) \\
=\int_{R^{2}}\left(\int_{R^{d-2}} \sum_{k, b}\left|s_{k, b}(f)\right|^{2} d \bar{x}\right)^{2} d \overline{\bar{x}} .
\end{aligned}
$$

Since $\mathscr{F}_{p} s_{k, b}^{a}(f)=\Psi^{a} \mathscr{F}_{p} s_{k, b}(f)$ and $\left\{\operatorname{supp} \Psi^{a}\right\}$ intersect finitely, the last term does not exceed

$$
C \int_{R^{2}}\left(\int_{R^{d-2}} \sum_{a} \sum_{k, b}\left|s_{k, b}^{a}(f)\right|^{2} d \bar{x}\right)^{2} d \overline{\bar{x}} .
$$

Thus a proof is complete. 
Proof of Sublemma. Fix $\bar{\xi}$ and $\bar{\eta}$ in $\boldsymbol{R}^{d-2}$. Then the sublemma follows from the fact that supp $\hat{s}_{k, b}(f)(\bar{\xi}, \cdot)+\operatorname{supp} \hat{s}_{l, c}(f)(\bar{\eta}, \cdot), b, c=0,1$, $\cdots$, are disjoint. In fact, supp $\hat{s}_{k, b}(f)(\bar{\xi}, \cdot)$ is contained in the annulus $\left\{\rho_{k}-5 \delta \rho_{k}^{-1} \leqq|\bar{\xi}| \leqq \rho_{k}+5 \delta \rho_{k}^{-1}\right\}$ and a disk of radius $<5 \delta^{1 / 2}$ centered at $\xi^{k, b}(\mathcal{C} p)$. Therefore a proof is reduced to the two dimensional case which is well known if $k=l$ (see Fefferman [6]). If $k \neq l$, we can prove it by a similar way with more careful computation.

4.2. Let $M \geqq N_{n} \geqq 1$ and $\varepsilon_{n}>0(n=1,2, \cdots)$ and $O_{n}$ be rotations in $\boldsymbol{R}^{d}$ which fix the first $d-2$ coordinates. Let $S_{n}=\left\{\left(\bar{x}, x_{d-2}, x_{d-1}\right) \in \boldsymbol{R}^{d}\right.$; $\left.|\bar{x}|<\varepsilon_{n} M,\left|x_{d-2}\right|<\varepsilon_{n} N_{n},\left|x_{d-1}\right|<\varepsilon_{n}\right\}$ and $R_{n}=O_{n} S_{n}$. For a function $f$ on $\boldsymbol{R}^{d}$ define $M_{n}(f)$ by

$$
M_{n}(f)(x)=\frac{1}{\left|R_{n}\right|} \int_{R_{n}} f(x-y) d y .
$$

Lemma 4. There exists a constant $C$ such that

$$
\int_{R^{2}}\left(\int_{R^{d-2}} \sum_{n}\left|M_{n}\left(f_{n}\right)\right|^{2} d x(p)\right)^{2} d x(\complement p) \leqq C(\log M)^{3} \int_{R^{2}}\left(\int_{R^{d-2}} \sum_{n}\left|f_{n}\right|^{2} d x(p)\right)^{2} d x(\complement p)
$$

for all $f_{n}$ in $\mathscr{S}\left(\boldsymbol{R}^{d}\right)$ and $M>2$.

Proof. Let $w$ be a non-negative function on $R^{2}$ and put

$$
I=\int_{\boldsymbol{R}^{2}} \int_{\boldsymbol{R}^{d-2}} \sum_{n}\left|M_{n} f_{n}(\bar{x}, \overline{\bar{x}})\right|^{2} w(\overline{\bar{x}}) d \bar{x} d \overline{\bar{x}} .
$$

By Schwarz's inequality

$$
\left|M_{n}\left(f_{n}\right)(\bar{x}, \overline{\bar{x}})\right|^{2} \leqq \frac{1}{\left|R_{n}\right|} \int\left|f_{n}(\bar{y}, \overline{\bar{y}})\right|{ }^{2} \chi_{R_{n}}(\bar{x}-\bar{y}, \overline{\bar{x}}-\overline{\bar{y}}) d \bar{y} d \overline{\bar{y}} .
$$

Substituting this inequality we have

$$
\begin{aligned}
& \int_{R^{d}} \mid M_{n} f_{n}(\bar{x}, \overline{\bar{x}})^{2} w(\overline{\bar{x}}) d \overline{\bar{x}} d \overline{\bar{x}} \\
& \quad \leqq \int\left|f_{n}(\bar{y}, \bar{y})\right|^{2} d \bar{y} d \overline{\bar{y}} \frac{1}{\left|R_{n}\right|} \int \chi_{R_{n}}(\bar{x}-\bar{y}, \overline{\bar{x}}-\overline{\bar{y}}) w(\overline{\bar{x}}) d \bar{x} d \overline{\bar{x}} .
\end{aligned}
$$

Put

$$
W(\overline{\bar{y}})=\sup _{n} \frac{1}{\left|R_{n}\right|} \int_{R^{2}}\left(\int_{R^{d-2}} \chi_{R_{n}}(\bar{x}, \overline{\bar{x}}) d \bar{x}\right) w(\overline{\bar{y}}+\overline{\bar{x}}) d \overline{\bar{x}} .
$$

Then the last integral is bounded by

$$
\int_{R^{2}}\left(\int_{R^{d-2}}\left|f_{n}(\bar{y}, \overline{\bar{y}})\right|^{2} d \bar{y}\right) W(\overline{\bar{y}}) d \overline{\bar{y}} \text {. }
$$

Thus 


$$
I \leqq \int\left(\int \sum_{n}\left|f_{n}(\bar{y}, \overline{\bar{y}})\right|^{2} d \bar{y}\right) W(\overline{\bar{y}}) d \overline{\bar{y}},
$$

which does not exceed, by Schwarz's inequality,

$$
\left(\int\left(\int \sum_{n}\left|f_{n}\right|^{2} d \bar{y}\right)^{2} d \overline{\bar{y}}\right)^{1 / 2}\left(\int W^{2}(\overline{\bar{y}}) d \overline{\bar{y}}\right)^{1 / 2} .
$$

Therefore our proof is complete if we show that

$$
\int W^{2} d \overline{\bar{y}} \leqq C(\log M)^{3} \int w^{2} d \overline{\bar{y}}
$$

with a constant independent of $M$ or $w$.

In fact, put

$$
\rho(\overline{\bar{x}})=\frac{1}{\left|R_{n}\right|} \int_{R^{d-2}} \chi_{R_{n}}(\bar{x}, \overline{\bar{x}}) d \bar{x}
$$

Since $O_{n}$ is a rotation which fixes the first $d-2$ coordinates,

$$
\rho(\overline{\bar{x}}) \leqq \frac{1}{4 \varepsilon_{n}^{2} N_{n}} \chi_{I_{n}}(\overline{\bar{x}})
$$

for some rectangle $I_{n}$ of size $2 \varepsilon_{n} \times 2 \varepsilon_{n} N_{n}$. Therefore

$$
W(\overline{\bar{y}}) \leqq \sup _{I} \frac{1}{|I|} \int_{I} w(\overline{\bar{y}}+\overline{\bar{x}}) d \overline{\bar{x}}
$$

where the sup runs over all rectangles $I$ in $R^{2}$ of eccentricity $\leqq M$. Thus (4.3) follows from Cordoba's theorem ([4]).

REMARK 3. Let $R_{n}^{\mu}$ be the set obtained from $R_{n}$ expanding by the factor $2^{\mu}$ and $M_{n}^{\mu}$ be the operator $M_{n}$ defined by the set $R_{n}^{\mu}$. Our proof shows that Lemma 4 holds if $M_{n}\left(f_{n}\right)$ is replaced by $\sum_{\mu=0}^{\infty} 2^{-\mu} M_{n}^{\mu}\left(f_{n}\right)$, in which form we apply it later.

4.3. Let $P_{n}=\left\{\bar{\xi}=\left(\xi_{d-2}, \xi_{d-1}\right) ; n \delta^{1 / 2} \leqq \xi_{d-1}<(n+1) \delta^{1 / 2}\right\}$ be strips in $\boldsymbol{R}^{2}$ and for $f \in \mathscr{S}\left(\boldsymbol{R}^{d}\right) f_{n}$ be the projection defined by

$$
\mathscr{F}_{\mathrm{C} p} f_{n}=\chi_{n} \mathscr{F}_{\mathrm{C} p} f \text {, }
$$

where $\chi_{n}$ is the characteristic function of $P_{n}$.

LEMMA 5. We have

$$
\int_{R^{2}}\left(\int_{R^{d-2}}|s(f)|^{2} d x(p)\right)^{2} d x(\complement p) \leqq C\left(\log \delta^{-1}\right)^{3} \int_{R^{2}}\left(\int_{R^{d-2}} \sum_{n}\left|f_{n}\right|^{2} d x(p)\right)^{2} d x(\complement p)
$$

for $f$ in $\mathscr{S}\left(\boldsymbol{R}^{d}\right)$, where $C$ is a constant independent of $f$ and $\delta$.

Proof. Let $t_{k}^{a}$ be the operator defined by 


$$
t_{k}^{a}(f)^{\wedge}(\xi)=\psi\left(\left(|\xi(p)|^{2}-\left(1-\rho_{k}^{2}\right)\right) / 3 \delta\right) \psi\left(\left|\xi(p)-\xi^{a}(p)\right| / 3 \delta\right) \hat{f}(\xi) .
$$

We remark that

$$
\boldsymbol{s}_{k, b}^{a}(f)=t_{k}^{a} \circ s_{k, b}^{a}(f) .
$$

In fact, if $\xi=(\bar{\xi}, \bar{\xi})=(\xi(p), \xi(\complement p)) \in \operatorname{supp} \hat{s}_{k, b}^{a}(f)$, then $-10 \delta<|\xi(p)|^{2}-$ $\left(1-\rho_{k}^{2}\right)<10 \delta$, which implies $\psi\left(\left(|\xi(p)|^{2}-\left(1-\rho_{k}^{2}\right)\right) / 3 \delta\right)=1$. Obviously $\psi\left(\left|\xi(p)-\xi^{a}(p)\right| / 3 \delta\right)=1$.

Since $t_{k}^{a}$ is defined by a multiplier depending only on the first $d-2$ variables $\xi(p)$ and since $\sum_{a} \sum_{k} \psi\left(\left(|\xi(p)|^{2}-\left(1-\rho_{k}^{2}\right)\right) / 3 \delta\right) \psi\left(\left|\xi(p)-\xi^{a}(p)\right| / 3 \delta\right)$ is uniformly bounded,

$$
\sum_{a} \sum_{k} \int_{R^{d-2}}\left|t_{k}^{a}\left(f_{n}\right)(\bar{x}, \overline{\bar{x}})\right|^{2} d \bar{x} \leqq C \int_{R^{d-2}}\left|f_{n}(\bar{x}, \overline{\bar{x}})\right|^{2} d \bar{x}
$$

for all $\overline{\bar{x}} \in \boldsymbol{R}^{2}$. Therefore by Lemma 3 it suffices to show that

$$
\int_{R^{2}}\left(\int_{R^{d-2}} \sum_{a} \sum_{k, b}\left|s_{k, b}^{a}(f)\right|^{2} d \bar{x}\right)^{2} d \overline{\bar{x}} \leqq C \int_{R^{2}}\left(\int_{R^{d-2}} \sum_{n, a, k}\left|t_{k}^{a}\left(f_{n}\right)\right|^{2} d \bar{x}\right)^{2} d \overline{\bar{x}} .
$$

Now $s_{k, b}^{a}(f)=\sum_{n} s_{k, b}^{a}\left(f_{n}\right)$. Since for each $b$ the support of $\Psi_{k, b}$ intersects the support of $\chi_{n}$ at most 11 times, we have $\sum_{b}\left|s_{k, b}^{a}(f)\right|^{2} \leqq$ $11 \sum_{n} \sum_{b}\left|s_{k, b}^{a}\left(f_{n}\right)\right|^{2}$. On the other hand for each $n$ the support of $\chi_{n}$ intersects the support of $\Psi_{k, 100 b}\left(b=0,1, \cdots, \delta^{-1 / 2} \rho_{k} / 200\right)$ at most a time. We denote such $a b$ by $b(n)$. Thus

$$
\sum_{a} \sum_{k, b}\left|s_{k, b}^{s}(f)\right|^{2} \leqq 11 \sum_{n, a, k}\left|s_{k, b(n)}^{a}\left(f_{n}\right)\right|^{2} .
$$

Next we remark that $s_{k, b(n)}^{a}\left(f_{n}\right)$ has a representation

$$
s_{k, b(n)}^{a}\left(f_{n}\right)=K_{k, b(n)} * t_{k}^{a}\left(f_{n}\right),
$$

where $\hat{K}_{k, b(n)}(\xi)=\Psi^{a}(\xi) \Psi_{k, b(n)}(\xi) \phi\left(\left(1-|\xi|^{2}\right) \delta^{-1}\right)$.

Assume for a moment $b(n)=0$. Then by an elementary calculus

$$
\left|K_{k, 0}(x)\right| \leqq C_{s, t, u} \delta^{d-1 / 2} \rho_{k}^{-1}|\delta \bar{x}|^{-s}\left|\delta \rho_{k}^{-1} x_{d-2}\right|^{-t}\left|\delta^{1 / 2} x_{d-1}\right|^{-u}
$$

for every $s, t, u \geqq 0$, where $C_{s, t, u} \leqq C \sum_{j=0}^{s+t+u}\left\|\phi^{(j)}\right\|_{\infty}$ with a constant $C$ independent of $\phi$.

Let $R_{k, 0}^{\mu}$ be a set such that $R_{k, 0}^{\mu}=\left\{\left(\bar{x}, x_{d-2}, x_{d-1}\right) \in \boldsymbol{R}^{d} ;|\bar{x}| \leqq 2^{\mu} \delta^{-1},\left|x_{d-2}\right| \leqq\right.$ $\left.2^{\mu} \rho_{k} \delta^{-1},\left|x_{d-1}\right| \leqq 2^{\mu} \delta^{-1 / 2}\right\}$ and $R_{k, b}^{\mu}=O_{k, b} R_{k, 0}^{\mu}$ where $O_{k, b}$ is a rotation such that $O_{k, b}(\bar{x}, 1,0)=\left(\bar{x}, \cos \delta^{1 / 2} \rho_{k}^{-1} b, \sin \delta^{1 / 2} \rho_{k}^{-1} b\right)$. Then $K_{k, b}$ is bounded by

Therefore

$$
C \sum_{\mu=0}^{\infty} 2^{-\mu} \frac{1}{\left|R_{k, b}^{\mu}\right|} \chi_{R_{k, b}^{\mu}}
$$

$$
\left|s_{k, b(n)}^{a}\left(f_{n}\right)\right| \leqq C \sum_{\mu=0}^{\infty} 2^{-\mu} \frac{1}{\left|R_{k, b(n)}^{\mu}\right|} \chi_{R_{k, b(n)}^{\mu}} *\left|t_{k}^{a}\left(f_{n}\right)\right| .
$$

By (4.5) and Lemma 4 with Remark 3 we have 


$$
\begin{aligned}
& \int_{R^{2}}\left(\int_{R^{d-2}} \sum_{a} \sum_{k, b}\left|s_{k, b}^{a}(f)\right|^{2} d \bar{x}\right)^{2} d \overline{\bar{x}} \leqq C \int_{R^{2}}\left(\int_{R^{d-2}} \sum_{n, a, k}\left|s_{k, b(n)}^{a}\left(f_{n}\right)\right|^{2} d \bar{x}\right)^{2} d \overline{\bar{x}} \\
& \leqq C\left(\log \delta^{-1}\right)^{3} \int_{R^{2}}\left(\int_{n, a, k}\left|t_{k}^{a}\left(f_{n}\right)\right|^{2} d \bar{x}\right)^{2} d \overline{\bar{x}}
\end{aligned}
$$

which completes a proof.

LEMMA 6. There exists a constant $C$ such that

$$
\int_{R^{2}}\left(\int_{R^{d-2}} \sum_{n}\left|f_{n}\right|^{2} d x(p)\right)^{2} d x(\complement p) \leqq C \int_{R^{2}}\left(\int_{R^{d-2}}|f|^{2} d x(p)\right)^{2} d x(\complement p)
$$

for all $f$ in $\mathscr{S}\left(\boldsymbol{R}^{d}\right)$.

Proof. Let $H=L^{2}\left(\boldsymbol{R}^{d-2}\right)$. Then the left hand side of (4.6) is written as

$$
\int_{R^{2}}\left(\sum_{n}\left\|f_{n}(\cdot, x(\complement p))\right\|_{H}^{2}\right)^{2} d x(\complement p p) .
$$

Now we apply an $H$-valued version of Carleson's theorem (cf. Rubio de Francia [10]) to get a bound of the above integral

$$
C \int_{R^{2}}\|f(\cdot, x(\mathfrak{C} p))\|_{H}^{4} d x(\mathfrak{C} p)=C \int_{R^{2}}\left(\int_{R^{d-2}}|f|^{2} d x(p)\right)^{2} d x(\mathfrak{C p}) \text {. }
$$

TheOREM 4. Let $0<\delta<1 / 4$. Then

$$
\int\left(\int|s(f)|^{2} d x(p)\right)^{2} d x(\complement p) \leqq C\left(\log \delta^{-1}\right)^{3} \int\left(\int|f|^{2} d x(p)\right)^{2} d x(\complement p)
$$

for $f \in \mathscr{S}\left(\boldsymbol{R}^{d}\right)$, where $C$ is a constant depending only on $d, \phi$ and $\psi$, more precisely $C \leqq C_{d, \psi} \sum_{j=0}^{d+1}\left\|\phi^{(j)}\right\|_{\infty}$.

Now choose a function $\phi^{\prime}$ so that $\phi^{\prime} \in C^{\infty}(-\infty, \infty), \operatorname{supp} \phi^{\prime} \subset(1 / 4,1)$, $\phi^{\prime} \geqq 0$ and $\sum_{k=-1}^{\infty} \phi^{\prime}\left(2^{k} t\right)=1$ for $0<t<1$. Let $\varepsilon>0$ and put $\phi(t)=t^{\varepsilon} \phi^{\prime}(t)$. Define $s_{k}^{\varepsilon}(f)$ by

$$
\hat{s}_{k}^{\epsilon}(f)(\xi)=\left(1-|\xi|^{2}\right)^{\varepsilon} \phi^{\prime}\left((1-|\xi|) 2^{k}\right) \hat{f}(\xi)
$$

Then

$$
s^{\varepsilon}(f)=\sum_{k=-1}^{\infty} s_{k}^{\varepsilon}(f)
$$

By Theorem 4

$$
\int\left(\int\left|s_{k}^{\varepsilon}(f)\right|^{2} d x(p)\right)^{2} d x(\complement p) \leqq C k^{3} 2^{-4 \varepsilon k} \int\left(\int|f|^{2} d x(p)\right)^{2} d x(\complement p) .
$$

Summing over $k=-1,0,1, \cdots$ it follows

THEOREM 5. If $\varepsilon>0$, then

$$
\int\left(\int\left|s^{\varepsilon}(f)\right|^{2} d x(p)\right)^{2} d x(\complement p) \leqq C \int\left(\int|f|^{2} d x(p)\right)^{2} d x(\complement p)
$$


for $f \in \mathscr{S}\left(\boldsymbol{R}^{d}\right)$, where $C$ is a constant independent of $f$.

4.4. Let $P$ be the family of subsets $p$ of $\{0,1, \cdots, d-1\}$ such that card $(p)=d-2$. Since the operator $s^{\varepsilon}$ is rotation invariant, (1.1) implies that

$$
\left\|s^{\varepsilon}(f)\right\|_{(4,2 ; p)} \leqq C\|f\|_{(4,2 ; p)}
$$

for $f \in \mathscr{S}\left(\boldsymbol{R}^{d}\right)$ and $p \in P$. By duality

$$
\left\|s^{s}(f)\right\|_{(4 / 3,2: p)} \leqq C\|f\|_{(4 / 3,2: p)} .
$$

Applying Theorem 3 to (4.7) and then an interpolation theorem for multilinear operators (cf. [1]) we get

THEOREM 6. Let $\varepsilon>0$ and $2 d /(d+1) \leqq u \leqq 2$. Then

$$
\left\|s^{\varepsilon}(f)\right\|_{u} \leqq C\|f\|_{u}
$$

for all $f$ in $\mathscr{S}\left(\boldsymbol{R}^{d}\right)$ of the form $f_{0}\left(x_{0}\right) f_{1}\left(x_{1}\right) \cdots f_{d-1}\left(x_{d-1}\right)$.

5. Restriction problem of Fourier transform. We apply our interpolation theorem to a restriction problem of Fourier transform. By a theorem in Tomas [11], if $1 \leqq u<2(d+1) /(d+3)$,

$$
\left(\int_{S^{d-1}}|\widehat{f}(\xi)|^{2} d \sigma(\xi)\right)^{1 / 2} \leqq C\|f\|_{u}
$$

for $f \in \mathscr{S}\left(\boldsymbol{R}^{d}\right)$ where $d \sigma(\xi)$ is the surface element and $C$ is independent of $f$. The inequality fails for $u>2(d+1) /(d+3)$ but a simple argument shows that (5.1) holds for $1 \leqq u<2 d /(d+1)$ if the functions $f$ are radial.

THEOREM 7. If $d \geqq 2,1 \leqq u \leqq 2 d /(d+1)$ and $f$ is a function in $\mathscr{S}\left(\boldsymbol{R}^{d}\right)$ of the form $f(x)=f_{0}\left(x_{0}\right) f_{1}\left(x_{1}\right) \cdots f_{d-1}\left(x_{d-1}\right)$, then

$$
\left(\int_{S^{d-1}}|\hat{f}(\xi)|^{2}\left|\xi_{0} \xi_{1} \cdots \xi_{d-1}\right|^{1 / d} d \sigma(\xi)\right)^{1 / 2} \leqq \frac{1}{\sqrt{2 \pi}}\|f\|_{u} .
$$

Proof. We assume $d>2$ but a careful reading shows that our proof applies to the case $d=2$. Let $w, f \in \mathscr{S}\left(\boldsymbol{R}^{d}\right)$ and assume supp $w(\xi) \subset$ $\left\{\left|\xi_{0}\right|>0\right\}$. By Fubini's theorem

$$
\int_{S^{d-1}}\left|\xi_{0}\right|^{1 / 2} \widehat{f}(\xi) w(\xi) d \sigma(\xi)=\int_{R^{d}} f(x) S w(x) d x
$$

where

$$
S w(x)=\frac{1}{\sqrt{2 \pi^{d}}} \int_{S^{d-1}}\left|\xi_{0}\right|^{1 / 2} w(\xi) e^{-i x \xi} d \sigma(\xi) .
$$

Therefore our problem reduces to an estimate of the following integral; 


$$
\begin{aligned}
& \int_{R^{d-1}}|S w(x)|^{2} d x_{1} \cdots d x_{d-1} \\
& \quad=\frac{1}{(2 \pi)^{d}} \int_{R^{d-1}} d x_{1} \cdots d x_{d-1} \int_{S^{d-1 \times S^{d-1}}}\left|\xi_{0} \eta_{0}\right|^{1 / 2} w(\xi) \bar{w}(\eta) e^{-i(\xi-\eta) x} d \sigma(\xi) d \sigma(\eta) .
\end{aligned}
$$

We introduce the polar coordinates: $\xi_{1}=\cos \theta_{1}, \xi_{2}=\sin \theta_{1} \cos \theta_{2}, \xi_{3}=$ $\sin \theta_{1} \sin \theta_{2} \cos \theta_{3}, \cdots, \xi_{d-1}=\sin \theta_{1} \cdots \sin \theta_{d-2} \cos \theta_{d-1}, \quad \xi_{0}=\sin \theta_{1} \cdots \sin \theta_{d-2} \sin \theta_{d-1}$. Then the last integral is transformed to

$$
\begin{array}{r}
\int_{S^{d-1}} d \sigma(\eta) \int_{R^{d-1}} d x_{1} \cdots d x_{d-1} \int_{D} \omega(\theta, \eta) \exp -i\left[\left(\xi_{1}-\eta_{1}\right) x_{1}\right. \\
\left.+\cdots+\left(\xi_{d-1}-\eta_{d-1}\right) x_{d-1}\right] d \theta,
\end{array}
$$

where $\omega(\theta, \eta)=\left|\xi_{0} \eta_{0}\right|^{1 / 2} w(\xi) \bar{w}(\eta) \prod_{j=1}^{d-1} \sin ^{d-j-1} \theta_{j} \exp \left[-i\left(\xi_{0}-\eta_{0}\right) x_{0}\right]$ and $D$ is the image of $S^{d-1} \cap \operatorname{supp} w$ by the mapping of $\xi$ to $\theta$.

Now fix $\eta$ and introduce new variables

$$
\begin{aligned}
\rho_{1}= & \cos \theta_{1}-\eta_{1}, \\
\rho_{2}= & \sin \theta_{1} \cos \theta_{2}-\eta_{2}, \\
& \cdots \cdots \cdot \sin \theta_{1} \sin \theta_{2} \cdots \sin \theta_{d-2} \cos \theta_{d-1}-\eta_{d-1} .
\end{aligned}
$$

Consider the Jacobian $|\partial \rho / \partial \theta|=\left|\sin ^{d-1} \theta_{1} \sin ^{d-2} \theta_{2} \cdots \sin \theta_{d-1}\right|$. The inner integral of (5.5) is transformed to

$$
\int_{R^{d-1}} d x_{1} \cdots d x_{d-1} \int_{\Delta} \omega(\theta, \eta) /\left|\frac{\partial \rho}{\partial \theta}\right| \exp \left[-i\left(\rho_{1} x_{1}+\cdots+\rho_{d-1} x_{d-1}\right)\right] d \rho_{1} \cdots d \rho_{d-1},
$$

where $\Delta$ is the image of $D$ by the mapping of $\theta$ to $\rho . \omega(\theta, \eta) /|\partial \rho / \partial \theta|$ is infinitely differentiable in $\rho$ since $w(\xi)$ vanishes near $\xi_{0}=0$. Therefore by Fourier inversion formula the last integral equals

$$
(2 \pi)^{d-1} \omega(\theta, \rho) /\left|\frac{\partial \rho}{\partial \theta}\right|
$$

at $\rho=0$. Since $\xi=\eta$ if $\rho=0$, the last term coincides with

$$
(2 \pi)^{d-1}\left|\xi_{0}\right||w(\xi)|^{2} /\left|\sin \theta_{1} \sin \theta_{2} \cdots \sin \theta_{d-1}\right|=(2 \pi)^{d-1}|w(\xi)|^{2} \text {. }
$$

Thus by (5.4) and (5.5)

$$
\int_{R^{d-1}}|S w(x)|^{2} d x_{1} \cdots d x_{d-1}=\frac{1}{2 \pi} \int_{S^{d-1}}|w(\xi)|^{2} d \sigma(\xi)
$$

Applying the reversed Hölder's inequality to (5.3) we get

$$
\left(\int_{S^{d-1}}\left|\xi_{0}\right||\hat{f}(\xi)|^{2} d \sigma(\xi)\right)^{1 / 2} \leqq \frac{1}{\sqrt{2 \pi}} \int_{R}\left(\int_{R^{d-1}}|f|^{2} d x_{1} \cdots d x_{d-1}\right)^{1 / 2} d x_{0},
$$

from which we have 


$$
\left(\int_{S^{d-1}}\left|\xi_{j}\right||\hat{f}(\xi)|^{2} d \sigma(\xi)\right)^{1 / 2} \leqq \frac{1}{\sqrt{2 \pi}} \int_{R}\left(\int_{R^{d-1}}|f|^{2} d x_{1} \cdots d x_{j-1} d x_{j+1} \cdots d x_{d-1}\right)^{1 / 2} d x_{j}
$$

Let $P$ be the set of $d-1$ indices in $\{0,1, \cdots d-1\}$ and $I_{p}, p \in P$, be disjoint arcs in $\partial D$ of length $2 \pi / d$. Let $\delta_{p}(z)$ be functions in the Hardy class $H^{2}(\boldsymbol{D})$ such that $\operatorname{Re} \delta_{p}\left(e^{i \theta}\right)=1$ a.e. in $I_{p}$ and $=0$ a.e. in $\partial \boldsymbol{D}-I_{p}$, and $\operatorname{Im} \delta_{p}(0)=0$. Then $\delta_{p}(0)=1 / d$. Identify $p$ with $j$ such that $j \notin p$ and define a mapping $T^{z}$ by

$$
T^{z} f(\xi)=\widehat{f}(\xi) \prod_{j=0}^{d-1}\left|\xi_{j}\right|^{\delta_{j}(z) / 2} .
$$

Applying Theorem $1^{\prime}$ with $\bar{M}=\boldsymbol{R}^{d}$ and $N=S^{d-1}$ we get Theorem 7 .

REMARK 4. If (5.2) is valid for every $f$ in $\mathscr{S}\left(\boldsymbol{R}^{d}\right)$, then we have

$$
\left(\int_{S^{d-1}}|\hat{f}(\xi)|^{2} d \sigma(\xi)\right)^{1 / 2} \leqq C\|f\|_{2 d /(d+1)}
$$

for $f$ such that supp $\hat{f} \subset\{\xi ;|\xi /| \xi|-(1,1, \cdots, 1) / \sqrt{d}|<1 / 2 \sqrt{d}\}$. Thus by rotation (5.6) holds for all $f$ in $\mathscr{S}\left(\boldsymbol{R}^{d}\right)$, which contradicts the optimality of Tomas's condition.

\section{REFERENCES}

[1] J. BERG AND J. LÖFSTRöm, Interpolation Spaces, An Introduction, Springer-Verlag, Berlin, Heidelberg/New York, 1976.

[2] L. Carleson and P. SJölin, Oscillatory integrals and multiplier problem for the disk, Studia Math. 44 (1972), 287-299.

[3] R. Coifman, M. Cwikel, R. Rochberg, Y. Sagher and G. Weiss, A theory of complex interpolation for families of Banach spaces, Adv. in Math. 43 (1982), 203-229.

[4] A. CoRdoba, A note on Bochner-Riesz operators, Duke Math. J. 46 (1979), 505-511.

[5] C. Fefferman, The multiplier problem for the ball, Ann. of Math. 94 (1971), 330-336.

[6] C. Frfferman, A note on spherical summation multipliers, Israel J. Math. 15 (1973), 44-52.

[7] J. Garnetr, Bounded Analytic Functions, Academic Press, New York/London, 1981.

[8] C. Herz, On the mean inversion of Fourier and Hankel transforms, Proc. Nat. Acad. Sci. U.S.A. 40 (1954), 996-999.

[9] L. HörmANDER, Oscillatory integrals and multipliers on $F L^{p}$, Ark. för Mat. 11 (1973), $1-11$.

[10] J.L. Rubio DE Francia, A Littlewood-Paley inequality for arbitrary intervals, Inst. Mittag-Leffler, rep. no. 18, 1983.

[11] P. A. Tomas, A restriction theorem for the Fourier transform, Bull. Amer. Math. Soc. 81 (1975), 477-478.

[12] A. ZYGMUND, On Fourier coefficients and transforms of functions of two variables, Studia Math. 50 (1974), 189-201.

Mathematical Institute

TÔHOKU UNIVERSITY

SENDAI 980

JAPAN 